Recepción: 31/07/2015

Evaluación: 10/08/2015

Aprobación: 25/09/2015

Artículo de Investigación Científica

\title{
MODELO DE GESTIÓN ESTRATÉGICO Y SISTÉMICO PARA LAS ENTIDADES EDUCATIVAS COMO ORGANIZACIONES INTELIGENTES
}

\author{
Ignacio Abdón Montenegro Aldana \\ Universidad Santo Tomas de Aquino Bogotá. \\ Grupo Investigación: Organización y Gestión Educativa. \\ ignacio.montenegro.aldana@gmail.com
}

Ángela Patricia Cárdenas Guerrero Universidad Santo Tomas de Aquino Bogotá. Grupo Investigación: Organización y Gestión Educativa angelapatriciacardenasguerrero@gmail.com

Maricel Cabrera R. Universidad Santo Tomas de Aquino Bogotá Grupo Investigación: Organización y Gestión Educativa Ministerio de Educación Nacional Colombia maricelcabrera@gmail.com

\begin{abstract}
RESUMEN
Este artículo presenta posibilidad de configurar un modelo de gestión estratégico y sistémico para las entidades educativas como organizaciones inteligentes. Lo estratégico se relaciona con la articulación de estructuras organizacionales y su propia gestión para garantizar fines de una entidad. Lo sistémico trata de identificar las instancias organizativas y sus relaciones entre sí para desarrollar una gestión de alto nivel que garantice el cumplimiento de su función. El artículo toma como base el estado del arte de una investigación en curso, donde tiene por objeto caracterizar seis secretarías de educación en Colombia de entidades territoriales certificadas (ETC), para administrar y ofrecer el servicio público educativo. Es la primera fase de un proyecto a mediano plazo, en la segunda fase se diseñará un modelo de gestión y en la tercera, la validación de este modelo.
\end{abstract}


La metodología empleada es de contrastación teórica con reorganización conceptual plasmada en la estructura del documento.

Finalmente se dice que la educación es un derecho de las personas que garantiza el Estado y se cumple a través de un sistema educativo el cual lo administra la entidad territorial certificada, por ello, el fin es crear un modelo de gestión estratégico y sistémico que permita potenciar la inteligencia de estas entidades; en estos últimos años las Secretarias de Educación implementaron proceso de Modernización que estableció el Ministerio de Educación Nacional, porque hubo falta de organización, de gestión, de eficiencia y de pertinencia en el desarrollo de los procesos y subprocesos internos y externos, asimismo permitió organizar la planta con sus respectivos perfiles y funciones, este proceso ayudó en alguna medida a obtener mayor organización y gestión.

Palabras clave: Modelos de gestión educativa, modelo estratégico, modelo sistémico, entidades educativas, organizaciones inteligentes.

\title{
STRATEGIC AND SYSTEMIC MANAGEMENT MODEL FOR EDUCATIONAL INSTITUTIONS AS INTELLIGENT ORGANIZATIONS
}

\begin{abstract}
This article presents the possibility of setting up a strategic and systemic management model for educational institutions as intelligent organizations. The strategy relates the articulation of organizational structures and their management to ensure the end entity. The article aims to identify organizational instances and their relationships with each other in order to develop high level management that ensures the fulfilment of its course function. The study takes as a basis state-of-theart of research, intended to characterize six Secretaries of Education in Colombia, certified territorial entities (ETC) to manage and provide public educational services. It is the first phase of a project in the medium term; in the second phase, the design of the management model and in the third the validation of this model. The methodology employs a theoretical comparison with conceptual reorganization, reflected in the structure of the document.

Finally, Education is the right of individuals guaranteed by the State and met through an educational system which manages its certification; the purpose of this territorial entity is to create a strategic and systematic management model, allowing the intelligent enhancement of these entities. In recent years the Secretariat of Education implemented a modernization process that led to the establishment of the National Ministry of Education because there was a lack of organization, manage-
\end{abstract}


ment, efficiency and relevance in the internal and external development processes and in the internal and external sub-processes while also allowing organization of administrative staff with its respective profiles and functions, a process that, to some extent, helped to obtain greater organization and management.

Keywords: Models of Educational Management, Strategic Model, Systems Model, Educational institutions, Smart Organizations.

\title{
MODELO DE GESTÃO ESTRATÉGICA E SISTÊMICA PARA INSTITUIÇÕES EDUCACIONAIS COMO ORGANIZAÇÕES INTELIGENTES
}

\begin{abstract}
RESUMO
Este artigo apresenta possibilidade de criação de um modelo de gestão estratégica e sistemico para para organizações educativas como organizações inteligentes. questões estratégicas relacionadas com a articulação de suas próprias estruturas e gestão organizacionais para garantir o fim de uma entidade. 0 sistêmico procura identificar corpos organizacionais e suas relações uns com os outros para desenvolver uma gestão de alto nível para garantir o cumplimento de sua função. 0 artigo baseia-se no estado da arte de uma investigação em curso, que tem como objetivo caracterizar, de educação na Colômbia entidades territoriais certificadas (ETC), para administrar e oferecer o serviço público educacional. Esta é a primeira fase do projecto, a médio prazo, na segunda fase de um modelo de gestão será concebido e na terceira, a validação deste modelo. A metodologia é contraste teórico com a reorganização conceitual refletido na estrutura do documento.

Finalmente, disse que a educação é um direito das pessoas garantida pelo Estado e é cumprida através de um sistema educacional que administra a entidade territorial certificada, portanto, o objectivo é criar um modelo de gestão estratégica e sistêmica, que permite melhorar a inteligência dessas entidades; nos últimos anos, os Ministérios da Educação implementaram processo de modernização que estabeleceu o Ministério da Educação, Nacional , porque houve uma falta de organização, de gestão, de eficiência e pertinência para o desenvolvimento de processos e segmentos internos e externos, também permitiu a organizar a planta com seus respectivos perfis e funções, este processo ajudou em alguma medida a uma maior organização e gestão
\end{abstract}

Palavras chave: modelos de gestão educacional , modelo estratégico, instituições de ensino, modelo sistêmicos , organizações innteligentes. 


\section{INTRODUCCIÓN}

El propósito de este artículo es examinar la posibilidad de configurar un modelo de gestión estratégico y sistémico para las entidades educativas como organizaciones inteligentes. Lo estratégico se relaciona con la articulación de las estructuras organizacionales y su propia gestión para garantizar los fines últimos de la entidad. Lo sistémico trata de identificar las instancias organizativas y sus relaciones entre sí para desarrollar una gestión del más alto nivel que garantice el cumplimiento de su función. Se asume la concepción teórica de que, una entidad educativa se comporta como organización inteligente, en la medida en que se conoce a sí misma, se comunica con el entorno y desarrolla un conjunto integrado de procesos para cumplir las metas, objetivos y finalidades relacionadas con su misión.

El artículo se basa en antecedentes investigativos y fácticos, junto con teoría validada sobre referentes conceptuales que permitan dilucidar, en primer lugar, la naturaleza de los procesos educativos. A partir de esta base se estudian las entidades educativas como organizaciones inteligentes que aprenden y el papel del conocimiento en ellas. Se dilucidan las implicaciones de una gestión educativa estratégica y sistémica para plantear los elementos básicos de un modelo para las entidades educativas a fin de que cumplan cabalmente con su misión de garantizar el derecho a la educación.

El artículo ha tomado como base el estado del arte de una investigación en curso, la cual tiene por objeto caracterizar seis (6) secretarías de educación en Colombia de entidades territoriales certificadas para administrar y ofrecer el servicio público educativo. Es la primera fase de un proyecto a mediano plazo, cuya segunda fase será el diseño propiamente del modelo de gestión y la tercera, la validación de este modelo.

\section{Metodología}

La estructuración del artículo toma como punto de partida la recolección de antecedentes fácticos, esto es la descripción de hechos propios de la gestión educativa en distintas latitudes. Se tomó como base el proceso de modernización de las secretarías de educación en Colombia, la descripción de modelos de gestión como el de México y la caracterización de algunos sistemas educativos exitosos como los de Alemania, Francia y Ecuador.

En un segundo momento se recopilan antecedentes investigativos o estudios previos sobre entidades educativas como organizaciones inteligentes que aprenden, el papel del conocimiento en estas organizaciones, la gestión 
educativa estratégicos y sistémicos, y finalmente, los modelos de gestión con estas características para entidades educativas.

En un tercer momento se realiza un rastreo conceptual de los temas anteriores, antecedido por uno de carácter general: la naturaleza de los procesos educativos.

Los tres momentos se integran en las cinco grandes temáticas que conforman el artículo. Como puede observarse; si bien, hay una referencia a los hechos, la metodología empleada es de contrastación teórica con una posterior reorganización conceptual plasmada en la estructura del documento.

\section{Planteamiento de la temática}

Las entidades inteligentes son aquellas que interactúan con su entorno y toman las mejores decisiones para el cumplimiento de sus metas. Ello es posible si sus estructuras especializadas están lo suficientemente conectadas y sincronizadas para la acción, si manejan adecuadamente la información, apropian el conocimiento suficiente y se comunican con el entorno. En esta dinámica, las organizaciones inteligentes aprenden, se adaptan al medio y garantizar su supervivencia.

En teoría, las entidades educativas como organizaciones inteligentes pueden apropiar sus características lo cual les permite cumplir con sus funciones misionales en sumo grado. Entre las entidades educativas se encuentran las instituciones educativas y los entes que administran la educación, entidades objeto del presente análisis. Pero en la realidad, las entidades que administran el servicio público educativo no se comportan en forma pura como organizaciones inteligentes; así mismo, están en el extremo opuesto de estar completamente desarticuladas. Se puede decir que las entidades reales comportan ciertos rasgos de inteligencia.

La idea de crear un modelo de gestión estratégico y sistémico para las entidades educativas que administran la educación, es potenciar su organización, su inteligencia y su conocimiento para que puedan cumplir con su misión de la mejor forma posible.

\section{Desarrollo del tema}

El desarrollo del anterior planteamiento temático se inicia con un acercamiento conceptual a la naturaleza de los procesos educativos. A partir de esta base se estudian las entidades educativas como organizaciones inteligentes que aprenden y el papel del conocimiento en ellas. Se dilucidan las implicaciones de una gestión educativa estratégica y sistémica para plantear los elementos básicos de un modelo para las entidades educativas a fin de que cumplan cabalmente con su misión de garantizar el derecho a la educación. 


\section{NATURALEZA DE LOS PROCESOS EDUCATIVOS}

La educación es quizás, una de las formas que la especie humana ha ideado para reproducir los saberes ligados a la existencia; para crear y recrear la cultura. Surgió como un fenómeno natural de aprendizaje de las actividades asociadas a la supervivencia, luego se consolidó como mecanismo de reproducción de la cultura y, finalmente se constituyó en hecho jurídico en la medida en que las sociedades crearon Estados para regular sus actividades. La educación como hecho jurídico da cuenta de servicio y derecho. Es servicio, en la medida en que los Estados la garantizan a su población. En Colombia la educación es un servicio público que cumple una función social, bien sea ofrecida por el Estado o por los particulares. La contraparte del servicio es el derecho que le asiste a una población de gozar de la educación para desarrollarse como persona, como colectivo humano, para acceder a los bienes de la cultura y forjarse en un campo específico de la actividad humana.

Como lo asevera (Ramos, 2008) "Cuando se declara la educación como un servicio público, esto conlleva a que sea asequible a todos sin distinción de ninguna clase, en condiciones de igualdad y oportunidad; con garantía de continuidad para adaptarse progresivamente a los cambios sociales" (p. 73).

En virtud de lo anterior, en Colombia, se han venido constituyendo los siguientes elementos que forman parte del derecho a la educación: El acceso, como posibilidad real que han de tener las personas al sistema educativo formal y, de manera especial entre los 5 y 15 años con una obligatoriedad de un año de preescolar y nueve de básica. (Art.67 CPC). La permanencia, como la existencia de condiciones institucionales y familiares para que los niños permanezcan en el sistema educativo, lo cual implica; en primer lugar, la satisfacción de necesidades básicas por parte de la familia y condiciones institucionales propicias como calidad de los escenarios, ambiente social, calidad pedagógica y bienestar estudiantil, entre otros. La calidad como un conjunto de propiedades que hacen de la educación un servicio que satisface las necesidades de formación de la población y colma las expectativas de los usuarios. Una educación es de calidad en la medida en que cumple los fines previstos en la Ley (Art. 5 Ley 115 de 1994), el cual se establece en el Decreto Único Reglamentario del Sector Educación No 1075 de Mayo de 2015 (Men, Colombia 2015) y la oportunidad como ingresar al camino formal y recorrerlo en las edades previstas.

\section{LAS ENTIDADES EDUCATIVAS COMO ORGANIZACIONES INTELIGENTES QUE APRENDEN}

Para comprender esta concepción teórica de las entidades educativas como organizaciones inteligentes que aprenden, se inicia con la reseña de unos estudios de acercamiento al tema, incluida la experiencia colombiana. Con esta base se precisa una síntesis teórica sobre el tema: 


\subsection{Estudios de acercamiento a las organizaciones inteligentes}

En distintas latitudes existen estudios sobre las organizaciones como entidades inteligentes, algunos de ellos generales y otros específicos sobre las instituciones educativas, los cuales merecen especial atención.

La tesis "Organizaciones inteligentes, una necesidad para las empresas modernas venezolanas, como aspecto clave para sustentar el rendimiento de la inversión" responde a la investigación de Anzola (2007). La intencionalidad del autor, es en primer lugar, describir la gestión de conocimiento en las empresas modernas venezolanas, con el propósito de conocer las acciones que orientan el mejoramiento sostenido en el desempeño. En segundo lugar, potenciar las ventajas competitivas de las empresas venezolanas, con el fin de orientar y aumentar el rendimiento de sus inversiones. Como aporte plantea, que nunca antes, las organizaciones habían enfrentado tantos retos, ni había existido la necesidad de que los lideres sintieran que no tenían las respuestas a muchos inconvenientes suscitados al interior de las organizaciones. Lo que en estos momentos abunda, plantea el autor, son las incertidumbres y las complejidades dentro de las organizaciones modernas.

La tesis, de Tintoré (2010) España, hace alusión a "las Universidades como organizaciones que aprenden: el caso de la Facultad de Educación de la Universidad Internacional de Cataluña". La autora presenta algunas consideraciones con respecto al concepto aprendizaje organizativo (A0), enfatiza en el proceso que tienen las organizaciones: aprenden, se adaptan, cambian y se desarrollan. A lo largo del estudio, plantea como mejoran sus aprendizajes y se analiza el caso concreto de la Facultad de Educación. En sus hallazgos corrobora, que todas las organizaciones aprenden y se adaptan al cambio, independientemente de los métodos que se ejecuten.

El sistema educativo en Francia, se enmarca desde el preámbulo de la Constitución francesa de 1946 que estipula "la Nación garantiza la igualdad de acceso, para los menores y los adultos, a la instrucción, la formación profesional y la cultura" Constitución Política Francesa (1946). El sistema comprende un derecho a la escolarización desde los 3 años y una escolarización obligatoria y gratuita para los niños de 6 a 16 años. La ley de orientación y programación para el futuro de la Enseñanza de 23 de abril de 2005, renovó las misiones al sistema educativo, entre ellas "garantizar la igualdad de oportunidades y conseguir que todos los alumnos concluyan con éxito sus estudios con la adquisición de una base común de conocimientos (dominio de la lengua francesa, matemáticas, práctica de un idioma extranjero, tecnologías de la información y la comunicación, cultura humanista) y favorecer la inserción profesional de los jóvenes". La France á la Loupe (2007), 1.

El sistema educativo francés permite la descentralización y autonomía de los territorios, y comprende un conjunto de niveles articulados: El primer nivel, el segundo nivel, tercer nivel - enseñanza superior, la formación profesional inicial, y la enseñanza para los alumnos con necesidades educativas especiales. 
El primer nivel de educación, corresponde a la enseñanza básica, que abarca la educación infantil y la educación primaria. En este caso, entonces, la educación infantil desarrolla:

"La enseñanza preescolar (educación infantil), creada en 1881, se dirige a los niños de 3 a 6 años, la educación infantil escolariza a casi todos los niños de 3 años, en su mayoría en centros públicos. Con un doble objetivo educativo y pedagógico, permite que los niños aprendan a vivir en sociedad, conformen su personalidad y desarrollen el lenguaje. La educación infantil es el punto fuerte y específico del sistema educativo francés y contribuye a mejorar la eficacia de la educación primaria. Los docentes de educación infantil reciben la misma formación que los de primaria y pueden enseñar en todos los grados de primaria”. La France á la Loupe (2007), 2.

La enseñanza primaria es obligatoria para todos los niños, tanto franceses como extranjeros, a partir de los 6 años. Se imparte a los niños de edades comprendidas entre 6 y 11 años. Su finalidad es impartir los elementos básicos del conocimiento y el aprendizaje de la ciudadanía. La France á la Loupe (2007), 2.

El segundo nivel corresponde a la enseñanza secundaria y abarca el collège y el lycée. Para el caso del Collège, se encuentran "escolarizados los niños de 11 a 15 años durante cuatro cursos académicos: de 6ำ a 9o, según la nomenclatura francesa. Se le llama único porque, en principio, se imparte la misma enseñanza a todos los alumnos. El Diploma Nacional (título de graduado en educación secundaria) acredita el fin de esta etapa. Y para el caso del Lycée, están escolarizados los adolescentes de 15 a 18 años, durante los cursos que van de $2^{\circ}$ a último curso. Los liceos ofrecen una gran variedad de formaciones. Son de dos tipos: al final del liceo de enseñanza general y tecnológica se obtiene el título de bachillerato general, y al final del liceo profesional se obtiene el CAP, el BEP o el bachillerato profesional" La France á la Loupe (2007), 3.

El tercer nivel, corresponde a la enseñanza superior, en donde coexisten dos subsistemas: el primero, un sistema abierto, en el cual, la universidades admiten la mayoría de los estudiantes, sin selección previa; y ofrecen procesos de formación muy diversas. El segundo, un sistema selectivo con una capacidad controlada. En el cual, los estudiantes ingresa a través de examen o expediente y una entrevista. Allí se forman principalmente los futuros dirigentes y altos mandos, tanto para el Estado como para las empresas.

La formación profesional inicial, es una enseñanza concreta en relación con las empresas y oficios, para adquirir conocimientos y habilidades propios de un campo profesional. Se ofrece en El liceo profesional, durante dos años, al cabo de este ciclo (collège), se otorga un certificado de aptitud profesional (CAP) o un diploma de estudios profesionales (BEP). La formación profesional inicial permite que los alumnos adquieran rápidamente un título para su inserción en la vida laboral. 
La enseñanza para los alumnos con necesidades educativas especiales, se efectúa a través de un conjunto de modelos diferenciados para atender las diversas discapacidades.

Para concluir, el sistema educativo francés es descentralizado, desde el siglo XIX, a través del cual, las regiones han venido ganando autonomía, especialmente desde los años 80 del siglo anterior; sin embargo, el Estado sigue teniendo competencia para determinar los programas, las orientaciones pedagógicas, la contratación y la remuneración de los docentes. Los municipios se encargan del funcionamiento de los centros de educación primaria con relativa autonomía en su gestión.

\subsection{Antecedentes en Colombia}

Antes de 2004, la prestación del servicio educativo por parte de las Secretarías de Educación en Colombia, se llevaba a cabo desde la precariedad: tecnológica insuficiente, ausencia de indicadores de gestión, desperdicio de recursos por falta de mecanismos de optimización, y dispersión de la información. Un diagnóstico de todo el sector arrojó oportunidades de mejora que fueron ayudando a definir las estrategias que apoyarían más tarde los componentes iniciales de reingeniería de procesos, tecnología y sistemas de Información.

Fue así como, en el marco de la política de eficiencia del sector educativo, desde el año 2004 el Ministerio de Educación Nacional ha venido desarrollando el Proyecto de Modernización de Secretarías de Educación (PMSE), que busca implementar las herramientas necesarias para organizar y hacer más eficaz la administración del sector, mediante el desarrollo de proyectos de reingeniería y la implementación de sistemas de información, así como de la puesta en práctica de actividades de gente y cultura con el personal de las secretarías. El PMSE “... busca que las Secretarías de Educación, renueven su gestión y sus estructuras con procesos orientados al cumplimiento de su misión y con sistemas de información de soporte apropiados, con el fin de prestar un servicio educativo en condiciones eficientes, con transparencia y equidad" (MEN, 2014).

El proyecto estructura unos objetivos básicos para mejorar esa gestión, entre los cuales se tienen: "apoyar a las instituciones educativas que las entidades territoriales tienen a cargo; modernizar las herramientas actuales, con el fin de optimizar la utilización de los recursos de las Secretarías de Educación, buscando mejorar sus indicadores de cobertura y calidad; implementar estructuras organizacionales acorde con la misión de las Secretarías de Educación de manera que se amplíe la capacidad de apoyo a las instituciones educativas de las entidades territoriales certificadas; promover un cambio de 
mentalidad y actitud en las Secretarías de Educación, con el fin de responder a nuevos retos" (MEN, 2004: 1).

El proyecto cuenta con los siguientes componentes: procesos, sistemas de información, estructura organizacional, gente y cultura, actividades complementarias. Mediante procesos de asesoría el PMSEE se fue implementando en cada una de las entidades territoriales.

\subsection{Hacia una síntesis teórica sobre las organizaciones inteligentes}

Los anteriores elementos dan pie a plantear una síntesis teórica sobre las entidades educativas como organizaciones inteligentes:

Garantizar la educación como derecho y como servicio implica la existencia de estructuras educativas. El Estado dispone de un sistema educativo que, nivel nacional, traza las políticas y lineamientos, a nivel territorial, administra el servicio educativo y a nivel institucional lo ofrece. En cada una de estos niveles, es preciso analizar la estructura y funcionamiento desde la perspectiva de organizaciones inteligentes.

Las organizaciones inteligentes son aquellas que poseen las propiedades de una entidad inteligente cualquiera: perciben, a través de sensores; procesan la información en una estructura interna que posee conocimiento y actúan sobre el medio, a través de efectores. Para el caso de una entidad territorial que administra el servicio público educativo, la percepción está dada por toda la información de entrada que se interpreta a la luz del conocimiento existente. Esa información es aportada por los distintos usuarios: estudiantes, padres de familia, docentes, directivos docentes, autoridades educativas, comunicadores, investigadores y políticos, entre otros actores sociales; también recibe información proveniente de entidades oficiales o privadas. El procesamiento de la información de entrada, se realiza en función de sus intencionalidades, con el respaldo de los distintos dispositivos de información existente sobre el estado del sistema: información jurídica, financiera, académica, bases de datos, estadísticas; información relacionada sobre procesos y procedimientos; además información sobre los distintos campos de gestión derivados de los planes de gobierno y de la actividad inercial, propia de las actividades recurrentes. El procesamiento de toda esta información la hacen los funcionarios, organizados por dependencias.

Peter Senge (1992) define una organización inteligente como "aquella en la que los individuos son capaces de expandir su capacidad y de crear los resultados que realmente desean. Aquí las nuevas formas y patrones de pensamiento son experimentados, en donde las personas aprenden continuamente y en conjunto, como parte de un todo" (36). Las organizaciones inteligentes podrían definirse de manera sencilla como aquellas que aprenden, que facilitan el aprendizaje a 
todos sus miembros, que son capaces de modificar sus normas de actuación (escritas o no) para responder a las nuevas exigencias del medio.

Toda la actividad anterior va encaminada a actuar sobre el medio; así, la entidad territorial realiza una gran variedad de acciones para cumplir su función: celebra contratos, realiza movimientos financieros, construye y repara establecimientos, distribuye materiales didácticos, orienta y controla a las instituciones educativas y responde a sus usuarios. Cada una de estas acciones las realiza desde unidades estructurales especializadas.

En teoría, el talento humano está organizado por perfiles para responder a las funciones especializadas propias de cada una de las dependencias. Obedece a unas condiciones de ingreso, permanencia a través del desarrollo institucional y finalmente, el retiro.

Como puede observarse existe una alta complejidad en la organización de la entidad territorial, complejidad incrementada por el flujo de personal, la movilidad interna y el crecimiento propio de la entidad.

Según León (2003), para que las organizaciones tradicionales se constituyan en organizaciones inteligentes, es decir, desarrollen su capacidad de aprendizaje y gestionen el conocimiento, deben cambiar su visión respecto del trabajo, pasando de un enfoque instrumental a una visión que integra los beneficios intrínsecos, aquellos que guardan una mayor coherencia con las aspiraciones humanas y que no sólo se restringen a la satisfacción de las necesidades más básicas. Se requiere conocer, practicar e incorporar, sobre todo en los seres humanos que dirigen los niveles estratégicos de la organización las cinco disciplinas sistémicas. Para empezar en la práctica de estas disciplinas, se debe partir en primer lugar por adoptar un cambio en el paradigma a través del cual se entiende los "problemas" y se busca las "soluciones" de nuestras organizaciones en el mundo real. Dentro de la generación de una organización inteligente cabe destacar la gran importancia del aprendizaje tanto a nivel personal como grupal, los dos niveles de aprendizaje deben estar ligados. El aprendizaje individual, en cierto nivel, es irrelevante para el aprendizaje organizativo, los individuos pueden aprender todo el tiempo y sin embargo no existir aprendizaje organizativo. Pero si los equipos aprenden, se transforman en microcosmos para aprender a través de la organización. Los beneficios de estos microcosmos, apoyados mediante la simulación de negocios son: experiencia en afrontar las consecuencias de decisiones que pueden tener efectos no deseados, la posibilidad de explorar una diversidad de desafíos prefijados a la administración, la oportunidad de adoptar diferentes papeles en un equipo, experiencia en persuadir y negociar con colegas sobre sus diferentes visiones de una situación de negocios. 


\section{PAPEL DEL CONOCIMIENTO EN LAS ENTIDADES EDUCATIVAS COMO ORGANIZACIONES INTELIGENTES}

El conocimiento es una representación interna que la entidad inteligente se hace de la realidad. Esta representación interna se construye a través de la experiencia y sirve para interpretar las percepciones y tomar decisiones para poder actuar. Con el fin de abordar este enfoque, se parte de algunos estudios previos sobre el tema y luego se concreta teóricamente:

\subsection{Estudios previos sobre el conocimiento en las organizaciones inteligentes}

La gestión del conocimiento es crucial para las organizaciones inteligentes que aprenden, así lo muestran algunos estudios:

En Perú, el autor Sarasara (2010) plantea la tesis, “Gestión del conocimiento y liderazgo de clase mundial, para el desarrollo del capital intelectual en las organizaciones inteligentes", el autor se interesa por determinar si el liderazgo a través de la gestión del conocimiento contribuye en la efectividad de las organizaciones inteligentes, tomando en cuenta, si el dominio personal del líder influye en su relación con la toma de decisión. En las conclusiones el autor plantea cómo a través de la gestión del conocimiento, se contribuye de manera efectiva a que las organizaciones inteligentes sean más proactivas y más competitivas. Los gerentes de las pequeñas y medianas empresas, son conscientes sobre la necesidad de implantar programas de formación para el recurso humano.

Lara (2005) plantea que la relación entre los factores de los constructos que forman la dimensión Gestión del conocimiento es muy débil. Dentro de las características externas los aspectos procesos de planificación estratégica y la vigilancia del entorno externo están ligeramente relacionados, mientras que el constructo capacidades internas sólo existe la débil relación entre sistema de medición de la gestión y stocks de conocimiento.

Por tanto, realmente la gestión del conocimiento es u constructo que se debe recoger a través de las diferentes dimensiones consideradas, dado que cada una de ellas está midiendo dicho constructo desde perspectivas diferentes.

Marabotto (2005), desarrolla una tesis a partir de constatar que las instituciones educativas en general son organizaciones destinadas primordialmente a la gestión y transferencia del conocimiento. Sin embargo los equipos de gestión que las conducen, cualquiera sea su nivel y con diferentes particularidades, tienen profundas dificultades para gestionar el conocimiento necesario para la toma de decisiones en relación al gobierno de las mismas y al cumplimiento de su misión, implicando este hecho una aparente contradicción, dada, por un lado la formación científica de los actores, 
y por otro lado, la naturaleza del conocimiento. Por otra parte, los modos de gestión del conocimiento de los equipos de gobierno afectan la eficacia de su actuación como tales, su capacidad para potenciar el rendimiento del capital intelectual, y por tanto, la calidad global de las organizaciones educativas. La investigación, de carácter exploratorio y cualitativo, indaga en primer lugar la evolución y el estado actual del recientemente conformado campo disciplinar denominado Gestión del conocimiento, caracterizado por su complejidad, ambigüedad y dinamismo, la falta de fronteras claras, la polisemia imperante y la infinidad de planos posibles para su abordaje, propios de un territorio muy joven, todavía bastante inexplorado y por el momento pluridisciplinar. La mayoría de dichas investigaciones y aportaciones teóricas se refieren casi con exclusividad al mundo de la empresa, últimamente, comienzan a insinuarse algunos desarrollos referidos a la administración pública y a organismos no gubernamentales.

A partir de los resultados obtenidos de una investigación realizada por Genesi (2010), concluyó que las competencias laborales (entre éstas, las técnico-comerciales, administrativas) están involucradas en el proceso de formación del talento humano en las organizaciones educativas inteligentes de manera equitativa, es decir, integralmente sin darle mayor énfasis a alguna en específico.

Con respecto a los elementos de la gestión de calidad del talento humano se destacan: participación, contribución, responsabilidad y liderazgo; de los cuales prevalece la falta de liderazgo en los gerentes educativos, profundizar la responsabilidad ante la comunidad educativa y mayor participación de los involucrados en el hecho educativo. Se concluye que la capacidad de la gestión del gerente es técnica, analítica e informática para el trato con las personas y toma de decisiones y la solución de problemas

Sobre las competencias requeridas en el ámbito pedagógico, administrativo y comunitario, atienden mayoritariamente lo administrativo; dejando a un lado la atención de lo pedagógico y comunitario, por el desconocimiento y falta de experiencia en las funciones que cumplen.

En cuanto a los talentos y habilidades referidos a los personales, de relación y de dirección de equipos, los gerentes educativos en el nivel técnico comercial presentan el conocimiento básico, pero no lo llevan continuamente a la práctica debido a factores externos, como son: estructura organizacional centralizada y las dificultades de comunicación con los municipios lejanos.

En suma, se trata de formar al docente para desempeñarse como tal, implementando cada uno de los roles para ser orientados hacia la gestión de calidad del talento humano en las organizaciones educativas inteligentes. 


\subsection{Concreción teórica sobre el conocimiento en las organizaciones inteligentes}

Los anteriores estudios dan pie para organizar los elementos teóricos concernientes al papel del conocimiento en las organizaciones inteligentes:

Las organizaciones inteligentes apropian y generan conocimiento y lo utilizan para consolidarse, fortalecerse, crecer y adaptase al medio. El conocimiento surge de la información como reducción de incertidumbre (Dretske, 1987: 9-18), como registro de eventos. La información se organiza en estructuras de datos que permiten realizar interpretaciones e inferencias. De la información surge el conocimiento, como interpretación de la información, como significado que es otorgado por una entidad inteligente.

El conocimiento es vital para los seres humanos individuales y para las organizaciones. Los procesos de apropiación, creación, transferencia y aplicación del conocimiento han generado una rama de estudio denominada "gestión de conocimiento". Las siguientes visiones ofrecen un conjunto de elementos para comprender de qué se trata la gestión del conocimiento dentro de las organizaciones inteligentes:

Muñoz (2003) plantea que la capacidad de adaptación al cambio es esencial para la supervivencia de las organizaciones y el conocimiento, el elemento clave. Gestionar el conocimiento de la organización demanda modelos integradores que vayan más allá de proporcionar información a las personas. El primer paso es obtener la visión global del conocimiento de la compañía (Von Krogh, 2001), sólo entonces es posible determinar acciones para su creación y transferencia. Los resultados de las publicaciones desde la década de los 80, muestran un desfase entre la investigación aplicada, centrada en el desarrollo de herramientas para la gestión de conocimiento y la creación de modelos que sustenten conceptos y procesos. La inversión en recursos tecnológicos, por sí misma, para la gestión de conocimiento ha dado lugar a resultados marginales que no aportan ventaja competitiva a las organizaciones. Problemas básicos como la medición del conocimiento organizacional y el contexto de la organización siguen sin resolverse. Se propone un ciclo de Gestión de Conocimiento que se inicia en el diagnóstico, con la creación del modelo de gestión ajustado a las necesidades y recursos de la compañía. La retroalimentación sobre los resultados cierra el ciclo del conocimiento.

Para Arteche (2003), la transformación en organización inteligente, el desarrollo de competencias y el fortalecimiento de capacidades, así también las alternativas para la colaboración como los son la «ecología del conocimiento»; y las «comunidades de práctica» pueden colaborar con las organizaciones educativas a la hora de marcar rumbo hacia la visión de la organización inteligente que ubica al conocimiento en el centro de la gestión. 
Martínez (2000), analiza el concepto de gerencia de organizaciones inteligentes: centrado en tres factores esenciales seleccionados por el autor y que enfocan la capacidad gerencial de las organizaciones hacia el autoaprendizaje y la innovación del conocimiento:

Una estructura inteligente capaz de producir bienes, servicios y conocimientos con calidad y costos eficientes. Entorno ambivalente conectado a la estructura interna mediante procesos de realimentación que originan conocimiento nuevo. Un sistema de incentivos de aprendizaje y autoaprendizaje. Se analizan los tres factores señalados en relación con la calidad del sistema de educación institucional, la posibilidad de mejorar los sistemas de capacitación en las empresas y los sistemas de incentivos organizacionales. Finalmente se establecen algunas recomendaciones para el mejoramiento de las políticas gubernamentales, como es el papel del sistema universitario y la acción de la gerencia de organizaciones inteligentes en función de la capacidad para producir conocimiento y llegar a ser más competitivos (Fajardo: 2011).

Según Sánchez (2006), el abordar la gestión de información, la gestión tecnológica y la gestión de recursos humanos y mostrar la relación de cada una de ellas con la gestión del conocimiento nos permite plantear que no es posible implementar la gestión del conocimiento sin demás gestiones y por ende, aquellas organizaciones que desarrollen en gran medida la gestión de información, la gestión tecnológica y la gestión de recursos humanos podrán llevar a cabo de manera ventajosa un programa de gestión del conocimiento. La gestión del conocimiento requiere de la adecuada gestión de la información, del uso apropiado de las tecnologías de información y de una correcta y moderna gestión de los recursos.

Sañudo (2014), propone un modelo de gestión del conocimiento fundado en la idea de contar con un patrón resultado de abstraer sus principales componentes y las relaciones, para que en diversas instituciones puedan generar a partir de ahí, estrategias específicas contextualizadas. Advierte que no hay modelos únicos que expliquen todas las dimensiones de la heterogeneidad de los procesos, pero a partir de los resultados de la investigación, es posible proponer un modelo de gestión de conocimiento orientado al almacenaje, sistematización, distribución y uso del conocimiento con compromisos sociales y éticos comunes.

Las instituciones educativas y las secretarías de educación como estructuras organizacionales, desarrollan gestión, adquieren información del medio y de sí mismas; esta información organizada permite la apropiación de conocimiento. Así mismo, la dinámica de sus propios procesos genera nueva información de la cual se infiere nuevo conocimiento. Pero una es la capacidad de apropiar y generar conocimiento y otra, la capacidad para aplicarlo en sus procesos de gestión. 


\section{GESTIÓN EDUCATIVA ESTRATÉGICA Y SISTÉMICA}

Los tres puntos anteriores dan pie para analizar en detalle una gestión educativa estratégica y sistémica. Para ello se inicia con la reseña de algunos antecedentes para luego precisar los elementos teóricos centrales

\subsection{Estudios previos sobre gestión educativa estratégica y sistémica}

Los siguientes estudios aportan elementos sobre la gestión educativa estratégica y sistémica que desarrollan las entidades educativas inteligentes:

El artículo "Gerencia de organizaciones inteligentes" de Martínez (2000), plantea la necesidad apremiante de analizar el concepto de gerencia, en las organizaciones inteligentes y cómo estas apuntan hacia el auto-aprendizaje y la innovación del conocimiento. La investigación le permitió al autor, realizar algunas recomendaciones para mejorar las políticas, redefinir el rol del sistema universitario, gerenciar las organizaciones inteligentes, en función de producir nuevo conocimiento y ser más competitivos.

En un estudio realizado por Ferrer (2012), con el fin de identificar los procesos gerenciales que se llevan a cabo en las organizaciones educativas; se pudo constatar que los procesos gerenciales que se realizan en las escuelas técnicas de los municipios Maracaibo y San Francisco son la planificación, organización, dirección y control; aunque medianamente de acuerdo a los resultados obtenidos, pues quienes las dirigen aun no las entienden como organizaciones que prestan un servicio a la comunidad.

Así mismo, reconocen, como características de los procesos gerenciales que se dan cita en las instituciones estudiadas las siguientes: concepción sistémica, racionalidad, creatividad, carácter cíclico y flexibilidad, aún y cuando la tendencia de los resultados obtenidos apunta a que la gerencia de estas organizaciones educativas casi nunca se ve impregnadas por ellas.

De igual forma, se determinó que en las organizaciones educativas estudiadas, no se aplica el enfoque inteligente en la gestión de las mismas, por lo que no se pueden considerar organizaciones inteligentes, toda vez, que los resultados revelan que casi nunca el personal docente que ocupa cargo directivos, al momento de gerenciar las instituciones educativas, tiene presente las cinco disciplinas: dominio personal, modelos mentales, visión compartida, aprendizaje en equipo y pensamiento sistémico. Por consiguiente, se identificó la necesidad de que los procesos gerenciales planificación, organización, dirección y control se ejecuten eficientemente bajo un enfoque inteligente para elevar así la calidad educativa de estas instituciones. En consecuencia, generó una propuesta de lineamientos que le va a permitir a quienes gerencian las organizaciones educativas del nivel medio técnico contar con un soporte teórico que contribuya a fortalecer su gestión y a transformarlas en organizaciones inteligentes. 


\subsection{Elementos para una síntesis teórica de una gestión estratégica y sistémica}

La anterior descripción de la estructura organizacional de las secretarías de educación permite caracterizar su gestión educativa, la cual está orientada a garantizar sus objetivos misionales.

Como lo plantea Porras (2012):

La gestión educativa, desde un punto de vista ligado a la teoría organizacional, es entendida como un conjunto de procesos teórico-prácticos integrados horizontal y verticalmente dentro del sistema educativo para cumplir con los mandatos sociales. Es un saber capaz de ligar conocimiento y acción, ética y eficacia, política y administración, en procesos que tienden al mejoramiento continuo de las prácticas educativas, a la exploración y explotación de todas las posibilidades y a la innovación permanente como proceso sistemático (36).

La gestión estratégica es una gestión prospectiva, que no solo administra lo que existe en el sistema, sino que configura visiones, planes y programas para ampliar el servicio educativo y mejorar su calidad y pertinencia a través del tiempo. Esta gestión estratégica se realiza a través de procesos: Los principales procesos objeto de la gestión, son justamente los misionales, orientados a garantizar el servicio público educativo para que la población en edad escolar acceda al sistema, permanezca en condiciones dignas y participe de una educación de calidad. Pero la función del Estado no es solo ofrecer el servicio sino regularlo; de ahí que, también son misionales, los procesos de inspección, vigilancia y supervisión, a través de los cuales, el Estado ejerce orientación, seguimiento, control y evaluación a las instituciones educativas oficiales y privadas para que ofrezcan el servicio de acuerdo con las normas jurídicas, técnicas y pedagógicas.

Los procesos misionales se sirven de los procesos de apoyo, que proveen condiciones institucionales propicias como la nómina, la gestión de personal y todos aquellos que contribuyen a forjar condiciones propicias para la gestión.

Desde otra forma de clasificación, se puede concebir una gestión misional expresada como gestión académica y gestión de comunidad. Habría una gestión de apoyo expresada en la gestión administrativa que incluye la directiva. En todo caso, la gestión estratégica articula todo tipo de gestión en torno a los ejes misionales.

El éxito de la gestión depende de la gerencia; esto es la forma como se dirigen las múltiples acciones para armonizarlas en torno de los propósitos institucionales. Se complementa con el liderazgo, la capacidad de armonizar toda la estructura organizacional, motivarla y movilizarla hacia las tareas centrales. La gerencia y el liderazgo en las organizaciones inteligentes reconocen el 
valor funcional de cada estructura orgánica, potencian su capacidad operativa, afinan de manera permanente los mecanismos de articulación y promueven de forma constante la unidad de propósitos.

La gerencia se ejerce especialmente en la administración de la entidad que, desde una visión clásica comprende los procesos de planeación, organización, seguimiento, control y evaluación desde un enfoque de auto-regulación. La planeación permite la previsión de acciones y recursos para lograr objetivos y metas a largo, mediano y corto plazo. De ahí la importancia de una planeación estratégica que permita visualizar el sistema educativo en el futuro y armonizar los planes de mediano y corto plazo para avanzar hacia ellos. Esta planeación general se desarrolla en los planes propios de cada área; cada una, se discrimina en los planes propios de cada unidad de trabajo, que a su vez, se desarrollan mediante la planeación propia de cada funcionario. En síntesis hay dos dimensiones de la planeación una temporal y otra estructural; la primera se hace a través de una línea de tiempo y la segunda dentro de los distintos niveles de la estructura organizacional.

La gerencia en los procesos de organización también tiene varios niveles: la organización "estándar" de la secretaría, la organización legal que es permanente y, una organización flexible que permite el desarrollo de los planes propios de cada obra de gobierno.

Los procesos de evaluación siguen la misma lógica de los de planeación, se apoya en el seguimiento; esto es el registro de los eventos significativos de todo el proceso de gestión. El control está asociado a los dos anteriores y tiene por objeto la intervención para corregir errores, dinamizar procesos con retardo o reorientar aquellos que se desvían de sus propósitos.

Lo fundamental de los distintos procesos inherentes a la administración es su efecto auto-regulador; la forma como se articula y se controlan unos a otros, en una dinámica compartida que le otorga cierta autonomía al sistema.

En la medida en que la estructura organizacional desarrolle su gestión desde una dinámica de auto-regulación, en esa misma forma, el sistema aprende: esto es, genera experiencia colectiva e incorpora conocimiento que lo usa para las nuevas acciones. Uno de los efectos del aprendizaje es el ajuste de la estructura organizacional para adaptarse al medio, para responder a las nuevas necesidades educativas.

Con respecto a la gestión educativa y sus relaciones con democracia y calidad, Sander (2012) plantea que "la construcción y reconstrucción del conocimiento en la educación y la gestión educativa comprometida con la calidad y la equidad implican un gran esfuerzo. Ese esfuerzo asume enormes proporciones en los países de América Latina, que necesitan multiplicar, de modo urgente, sus conocimientos científicos y tecnológicos para que puedan 
participar activamente y beneficiarse equitativamente de la transformación política y económica sin precedentes en el mundo moderno" (Pág. 9) Señala el autor la urgente necesidad de implementar la responsabilidad ciudadana y el espíritu público para construir y ejercer una administración colectiva que promueva una educación con identidad, equidad y calidad.

\section{MODELO DE GESTIÓN ESTRATÉGICO Y SISTÉMICO PARA LAS ENTIDADES EDUCATIVAS}

El desarrollo de los cuatro puntos anteriores permite abordar la posibilidad de diseñar modelos de gestión estratégicos y sistémicos para las entidades educativas como organizaciones inteligentes. Para ello se enuncian algunos antecedentes con el fin de plantear, aunque de manera primigenia, los elementos centrales de uno de estos modelos:

\subsection{Antecedentes sobre modelos de gestión de las entidades educativas}

Algunos estudios intentan delinear lo que sería un modelo de gestión de las organizaciones inteligentes:

Serlin (2010), en su tesis: "Conocimiento de la gestión de las organizaciones, sistemas complejos, dinámicos, inestables, adaptativos", describe procesalmente los cambios en la gestión, enfatiza en los diversos paradigmas que "oficialmente" son teorías administrativas repetitivas y que se reducen a la estandarización de procesos. Indagar sobre este aspecto, requiere que se mire desde otra perspectiva, para demostrar que ésta teoría adaptada a los modelos de gestión, se funda en los sistemas dinámicos y complejos.

Aguerrondo (1990), da a conocer un modelo de gestión para la escuela, pretende que las organizaciones resuelvan de manera más efectiva, los diferentes problemas, en la medida en que éstas van creciendo. La escuela como organización inteligente, practica la autorregulación, el dinamismo y la auto-evaluación: pilares fundamentales de su cultura organizacional. Dentro de sus conclusiones, plantea que la escuela latino-americana, no cuenta con el apoyo real, en cuanto a capacitación del personal, para que se logre una optimización de recursos y procesos.

En la tesis "Modelos de gestión basados en un enfoque de las organizaciones inteligentes para la Facultad de Ciencias Económicas y Sociales en Estados Unidos", Valecillos (2009), presenta los resultados de una propuesta, sobre un modelo de gestión basado en un enfoque de las organizaciones inteligentes, para dar respuesta a los requerimientos que demanda un currículo integral por competencias y hacer de la Facultad una organización de aprendizaje permanente. El autor evalúa en su sistema de gestión, los valores en los que se centra el desarrollo de la organización, el talento humano y los procesos de 
planificación, evaluación y seguimiento. Los aportes que hace a la comprensión del aprendizaje organizacional y de las capacidades de auto-organización, impulsa permanentemente a que las organizaciones sean innovadoras e intervengan de manera respetuosa y eficiente en los diversos subprocesos.

El sistema educativo de Alemania, en los últimos tiempos se consolida desde dos concepciones educativas con diferencias, la primera de carácter occidental del antiguo territorio de la República Federal de Alemania (RFA) y la segunda, propia del sistema pre comunista de la antigua República Democrática de Alemania (RDA), en el cual fue significativo el cambio. El sistema educativo público, lo maneja cada uno de los 16 estados federados (Lander): cada estado federal tiene su propio sistema educativo con ministerio de educación propio y el Estado Federal (Bund), fija los objetivos, las medidas y las orientaciones generales, que implementan los estados federados.

A pesar de la distribución diferenciada de competencias, los Länder y el Estado Federal, cooperan en determinadas actuaciones que son importantes para todo el ámbito territorial, como en la construcción de universidades, la planificación educativa, la promoción de instituciones y en proyectos de investigación científica de ámbito supraestatal. Es por ello que en La Constitución Federal Berengueras (2012: 2), como base fundamental de la legislación de cualquier estado o Land, recoge la libertad de la investigación y la enseñanza (artículo 5, párrafo 3), los derechos de los padres y madres (artículo 6, párrafo 2) y la competencia de los Länder en la supervisión de todo el sistema educativo de su ámbito territorial (artículo 7, párrafo 1). Berengueras (2012: $3)$.

El sistema educativo alemán se estructura en cinco niveles educativos preescolar (Kindergarten), escuela básica o primaria, secundaria de nivel I, secundaria de nivel II y, superior o sector terciario. En el Kindergarten, la modalidad más conocida y tradicional está dirigida a los niños y niñas entre los 3 y los 6 años. El Vorklassen (clases preescolares), fueron creadas en algunos Länder para niños y niñas de 5 años, de carácter no obligatorio. El Skchulkindergärten (Jardines de Infancia escolares) son para los niños y niñas que todavía no se encuentran preparados para entrar en la educación primaria, a pesar de tener cumplidos los 6 años de edad.

El siguiente nivel de educación obligatoria, comienza para todos los niños y niñas a los 6 años de edad y tiene un ámbito temporal, generalmente de doce años, los tres últimos considerados de escolaridad parcial obligatoria, en este sentido,

"La educación primaria inicia a los 6 años, y consta de cuatro cursos, excepto en los Länder de Berlín y Brandeburgo, en los que consta de seis cursos; su escolarización se lleva a cabo en los Grundschulen (escuelas de primaria), en este nivel, se enseña a leer, escribir y contar y, a partir de tercer curso, se inicia 
la lengua extranjera, por lo general la inglesa. Las materias más importantes son el alemán y las matemáticas, que son complementadas con las materias de música, religión y competencias sociales; al finalizar este periodo de primaria, no se realiza ningún examen final ni se otorgan diplomas o certificados de estudios, pero si reciben todos los alumnos un boletín de calificaciones; el paso de la enseñanza primaria a la enseñanza secundaria, es decir, de Grundschulen a cualquiera de los cuatro tipos de escuelas de secundaria existentes, está regulado de forma diferente en cada Land". Berengueras (2012), 5.

La educación secundaria se divide en dos niveles, la educación secundaria I, que comprende del quinto al décimo curso (en losLänder de Berlín y Brandeburgo, del séptimo al décimo curso) de los 10 o 12 años hasta los 16 años y, la educación secundaria II, que comprende todos los itinerarios escolares que tienen como base el grado anterior, de los 16 a los 19 años aproximadamente. Para Secundaria I Se imparte sobre la base de la enseñanza primaria común a todos los escolares y en la mayor parte de los Länder existen cuatro tipos de centros: Hauptschule (escuela general básica de enseñanza secundaria), tienen la función de transmitir una base amplia de cultura general que prepare al alumnado para su trayectoria profesional futura. La gran mayoría, al finalizar, continúan estudios en una escuela profesional. Los alumnos que superan todos los cursos obtienen el título de enseñanza secundaria básico (HauptschulabschulB) que facilita y permite el ingreso al sistema dual de formación profesional. Realschule (escuela secundaria donde se imparte una enseñanza de contenido más amplio), dedica menos atención a la formación profesional y más a las materias básicas de alemán, lenguas extranjeras y matemáticas. Los alumnos que se gradúan con éxito pueden pasar al ciclo superior del Gymansium y obtienen el título RealschulabschuB. Gymnasium (instituto de enseñanza secundaria media que incluye los estudios de bachillerato), tiene la función de impartir las materias a un nivel de rendimiento más elevado, porque su alumnado va a la Universidad. Gesamtschule (escuela comprensiva que ofrece más de un itinerario escolar). Berengueras (2012: 5).

Además, existen escuelas técnicas, que imparten formación profesional avanzada (Fachschule), que sirven para el perfeccionamiento profesional con una duración aproximada de uno a tres años académicos. La enseñanza terciaria, comprende los centros de enseñanza superior de carácter universitario (Hochschule), así como otras instituciones que ofrecen estudios de formación profesional a estudiantes del grado de secundaria II y habilidades para estudiar en escuelas superiores. Berengueras (2012: 10).

Alemania tiene un sistema de educación con niveles y ciclos para la atención de niños y jóvenes. Cada ciclo desde sus características propias, articula los procesos de gestión. Los Länder y el Estado Federal cooperan en determinadas 
actuaciones para favorecer el desarrollo de políticas en educación en de la nación.

El sistema educativo en Chile se caracteriza por la descentralización, iniciada en 1980. Los municipios se hacen cargo de la administración del servicio educativo; las políticas para asegurar la cobertura, la calidad y la pertinencia las imparte el Ministerio de Educación. El sector está organizado en los niveles educativos: prebásico, básico, medio y superior; los tres primeros son coordinadas por la unidad de currículo y evaluación que hace parte la subsecretaria de educación del Ministerio de Educación. En estos niveles se fomentan actividades y programas en el ámbito de formación en servicio y el perfeccionamiento de los docentes. Para el nivel de superior hay un Consejo Nacional de Educación Superior que organiza e implementa las políticas de educativas controla e inspecciona el sector y propende por el mejoramiento de la calidad. UNESCO (2010: 6-7).

En México, la Secretaria de Educación Pública tiene como propósito esencial, crear condiciones para favorecer el acceso a los niños, jóvenes y los adultos a una educación de calidad en los diversos niveles educativos. En esta perspectiva, la educación básica comprende los ciclos de formación de educación preescolar, primaria y secundaria. Para ello la educación preescolar, “atiende a niños de 3, 4 y 5 años de edad, se imparte generalmente en tres grados, no es un nivel obligatorio y para matricularse en el segundo o tercer grado no es necesario que haya cursado los anteriores. La mayor parte de los niños que entran a primaria han cursado, por lo menos, un grado preescolar, su objetivo es impulsar la creatividad y habilidades de los niños". Ceneval, México D.F. (2013: 3).

La educación primaria, es obligatoria y se imparte en seis grados, a partir de los 6 años. La educación secundaria, es obligatoria y se proporciona en tres años para las personas que culminan la primaria. Es necesaria para continuar con estudios medios profesionales o medios superiores. La educación media superior se le conoce como bachillerato o preparatoria y se cursa en un periodo de tres años, divididos generalmente en semestres. Para ingresar a este nivel es necesario contar con el certificado de la secundaria y regularmente se debe presentar también un examen de admisión. La educación superior se divide en estudios de grado y posgrado, su objetivo es formar profesionales. La mayor parte de las universidades públicas son autónomas de los gobiernos federal y estatal.

En la educación superior existen los institutos tecnológicos (ITES) que forman profesionales competentes con planes y programas de estudios acordes a la realidad de cada región. Este nivel se divide en ciclos como, institutos tecnológicos federales, institutos técnicos estatales. Existen distintos tipos de universidades: técnicas, tecnológicas, politécnicas, interculturales; también, educación a distancia entre otras. 
En México, entre los años 2001-2002, se implementó el Programa Escuelas de Calidad (PEC), para ello, la Escuela Pública Mexicana generó un modelo de gestión, basado en la autogestión y fundamentado en principios democráticos. La puesta en marcha del PEC, respondióa la política educativa que se implementó para transformar la gestión escolar en México. Desde entonces, este programa promueve un modelo de gestión escolar que impulsa el fortalecimiento de las entidades o secretarías de educación, los directores, maestros y padres de familia, para que decidan colectivamente la implementación de estos modelos de gestión para mejora la calidad de la educación. En este marco de gestión, se realiza y aplica un ejercicio de planeación estratégica. La planeación es necesaria para mejorar la gestión del sector de la educación y la escuela en los asuntos pedagógicos, organizativos, administrativos y de participación social, bajo el supuesto de que, si transforman sus prácticas y relaciones, entonces habrá mejores condiciones para mejorar el aprendizaje y los resultados educativos de todos los alumnos. El PEC, en coherencia con su enfoque dirigido a desarrollar las condiciones institucionales, que le permitan a la escuela gozar de una efectiva autonomía, se propuso ampliar los márgenes de confianza para administrar de manera directa los recursos que le ofrece el Programa y así financiar su Plan Estratégico.

El propósito del Programa Escuelas de Calidad (PEC) se ha centrado en contribuir al mejoramiento de los resultados educativos, a partir de la transformación de las formas de gestión de las escuelas, promoviendo la implementación de un modelo de gestión educativa estratégica basado en la capacidad para la toma de decisiones de manera corresponsable, en un liderazgo compartido que impulse el trabajo colaborativo, en una participación social responsable, con prácticas innovadoras que atiendan a la diversidad de los alumnos y a la evaluación para la mejora continua, a través de un enfoque estratégico. Vázquez \& otros (2010: 12).

Se pude afirmar que México implementa niveles educativos y ciclos de formación para que niños, jóvenes y adultos tengan acceso a la educción. Se implementa en las escuelas públicas un modelo de gestión estratégico y el Programa Escuelas de Calidad (PEC), el cual favorece los resultados educativos a partir de la trasformación de la gestión en las escuelas públicas de México. Sin embargo, en las entidades que administran la educación de México hay ausencia de modelos específicos de gestión.

En la subsecretaría de Educación Básica de México, según Castillo (2009) se diseña y se desarrolla una descripción del modelo estratégico de gestión educativa, ejecutado en el programa "escuelas de calidad", este tiene como propósito, mejorar el desempeño de las unidades administrativas de la SEP, mediante el control de procesos, alineación de estructuras organizacionales e implementación de modelos de dirección que contribuyen a mejorar la calidad educativa y la percepción ciudadana de los servicios que ofrece la Secretaría. Con el modelo se pretende minimizar la duplicación de tareas, generar mayor 
cohesión entre entidades federativas, organismos vinculados a la prestación de servicios y las escuelas. La política educativa de México se enfoca a establecer modelos de gestión que permitan la articulación entre los diversos entes y se fortalezcan las redes de aprendizaje entre los diferentes organismos.

El presente artículo, este modelo estratégico de gestión educativa aporta los elementos sustanciales por la unidad de propósitos, ya que se trata de mejorar el desempeño de las entidades territoriales mediante el control de procesos, la alineación de las estructuras organizativas y la implementación de un modelo de dirección que permita lo anterior. Los demás estudios referenciados, salvo uno, corresponden a entidades educativas del orden institucional, bien sean en la básica o en la superior. Éstos aportan valiosos elementos para comprender la organización de las entidades territoriales, en cuanto a calidad del talento humano, desarrollo organizacional, complejidad de la organización (especialmente en periodos de crecimiento) y la efectividad misma como organizaciones inteligentes en cuanto a pro actividad y competitividad. En el campo de la gestión, los estudios contribuyen a la comprensión de la gerencia de organizaciones inteligentes para orientar con liderazgo, los procesos de planificación, seguimiento, control y evaluación, desde un enfoque de autoregulación. Se hace necesario, además, el análisis de la dinámica que pueda existir entre los procesos estandarizados y los mecanismos de adaptación, propios de una entidad inteligente. En este contexto, se analiza el papel del conocimiento dentro de la organización inteligente, la forma como se apropia y se genera para dar sentido y efectividad a la gestión.

\subsection{Elementos centrales de un modelo de gestión estratégico y sistémico}

Para que las secretarías de educación se consoliden, se fortalezcan, crezcan y se adapten al medio; es necesario que apropien y generen conocimiento y lo utilicen para el mejoramiento de su gestión. Esto puede hacerse mejor desde un modelo.

Un modelo es la representación formal de un sistema. Para representar el sistema inherente en cada secretaría de educación se requiere la descripción de la estructura organizacional y la identificación de la dinámica de la gestión. Esto se hace mejor si se parte de un modelo ideal, un modelo orgánico funcional que de una parte, conciba la estructura organizacional como un conjunto articulado de instancias que obedece a una función con unidad de sentido. Y en segundo lugar, una estructura de la gestión que articule las diversas acciones con la misma unidad de sentido.

Valecillos (2009), propuso un modelo de gestión para la Facultad de Ciencias Económicas y Sociales de LUZ basado en el desarrollo de la organización y del talento humano, procesos de diagnóstico, planificación, evaluación y seguimiento del cambio y unas formas de intervención para el aprendizaje en 
los subsistemas: humano cultural, funcionamiento organizacional, tecnología de operación y el ambiente físico. El modelo coadyuva a superar las debilidades de la gestión signada por la burocracia, la lentitud, el autoritarismo, la ineficiente, la reproducción de lo que ya se sabe y la incapacidad para el aprendizaje organizacional. Enmarcan la acción gerencial en la representación global del sistema en las mentes de los individuos y su conectividad, facilitan la confianza y la seguridad que debe existir entre el personal e inducen a valorar la excelencia de las personas.

El Modelo de Gestión Educativa Estratégica (MGEE) de México surge con el propósito de apoyar a los centros escolares en la mejora de la calidad de los servicios que ofrecen, a partir del desarrollo de las competencias de todos los actores escolares para la práctica de liderazgo, trabajo en equipo colaborativo, participación social responsable, planeación estratégica, evaluación para la mejora continua, como asuntos claves de gestión, para enfrentar los retos globales del siglo XXI, en un marco de corresponsabilidad, transparencia y rendición de cuentas. En el centro del MGEE se encuentra la comunidad educativa. Los apoyos interdependientes y requeridos para la operación del MGEE son liderazgo compartido, trabajo en equipo colaborativo (trabajo colaborativo), participación social responsable, planeación estratégica y evaluación para la mejora continua (Sep. 2015).

El Nuevo Modelo de Gestión Educativa del Ecuador (NMGE) es un proyecto que inició su gestión en enero de 2010, y plantea la reestructuración del Ministerio de Educación para garantizar y asegurar el cumplimiento del derecho a la educación. Es decir, busca influir de manera directa sobre el acceso universal y con equidad a una educación de calidad y calidez, lo que implica ejecutar procesos de desconcentración desde la Planta Central hacia las zonas, distritos y circuitos, para fortalecer los servicios educativos y aproximarlos hacia la ciudadanía, atendiendo las realidades locales y culturales. (MEN Ecuador, 2015).

Con base en lo anterior un modelo de gestión es estratégico en la medida en que los integrantes de la estructura organizacional conciben y trabajan por una visión unificada y compartida. Es sistémico, si las diferentes instancias orgánicas se encuentran articuladas, comparten información, generan conocimiento y realizan los procesos en forma integrada para el cumplimiento de su misión.

\section{Conclusiones}

La educación surge como un hecho natural que se transforma en un hecho cultural y finalmente en un hecho jurídico. En este sentido se entiende como un derecho de las personas que garantiza el Estado. Para el cumplimiento de esta obligación, el Estado genera un sistema educativo el cual es administrado por 
entidades territoriales. Estas entidades poseen una estructura organizativa y una gestión para el cumplimiento de sus fines misionales. La idea de crear un modelo de gestión estratégico y sistémico es potenciar la inteligencia de estas entidades para que cumplan su función de la mejor manera posible.

La experiencia de democratización de los procesos de gestión en las escuelas y en las secretarías de educación, están relacionadas con el movimiento de modernización del país, éstas fueron objeto de un gran impulso renovador para el área de la educación, por los grandes cuestionamientos a nivel pedagógico que se suscitaron en el escenario público. La crítica al excesivo grado de centralismo administrativo, la rigidez en las estructuras organizacionales, la falta de autonomía y la precariedad en los sistemas de información, llevo a que las instituciones en general aprendan, se adapten, cambien y mejoren.

Hoy, se puede evidenciar que las Entidades Educativas, están permeadas por nuevos modelos de gestión. Estos paradigmas han impuesto novedosos campos de articulación y de consulta, lo que implica la consolidación de un proyecto Político-pedagógico para garantizar la "Educación como un derecho", en donde se debe garantizar el acceso, la permanencia y la calidad. La gestión de la escuela, busca el apoyo y la intermediación de las Secretarías de Educación y de la comunidad educativa para asegurar el cumplimiento de las exigencias legales, planteadas en la Constitución. Sin embargo, a pesar de todos los esfuerzos, la escuela pública se encuentra distante de cumplir éstas metas porque requiere de un presupuesto significativo para atender las necesidades de la comunidad.

Las organizaciones inteligentes tienen un enfoque claro de la calidad, consideran objeto de análisis y de cualificación a todas las personas y actividades de la organización, superando el tradicional formalismo de los formatos y requisitos. Existe un alto reconocimiento del factor humano, como elemento clave de la producción y de la satisfacción, tanto de los agentes externos como internos que intervienen. Los nuevos enfoques y perspectivas que manejan las entidades educativas, permiten vislumbrar, a pesar de sus limitaciones económicas y administrativas una evolución en el concepto de calidad, oportunidad y permanencia.

Las instituciones educativas, como organizaciones que aprenden, implementan un modelo de gestión específico, para optimizar sus procesos, buscan información del medio y de sí mismas, esta información organizada les permite apropiarse de un nuevo conocimiento, que será el insumo para optimizar y generar nuevos planteamientos y/o proyectos. Esta dinámica genera permanentemente nueva información, el reto está en contextualizar y aplicar lo innovador a los procesos de gestión que se llevan a cabo. 


\section{REFERENCIAS BIBLIOGRÁFICAS}

Aguerrondo, I. La escuela como organización inteligente. Buenos Aires. Argentina: Troquel, 1990.

Anzola, I. Organizaciones inteligentes, una necesidad para las empresas modernas venezolanas como aspecto daré para sustentar el rendimiento de la inversión. Venezuela (2007). Recuperado el 3 de marzo de 2015 de http://bibadm.ucla.edu.ve/edocs_baducla/tesis/P907.pdf

Arteche, M. Retos y alternativas de la gestión del conocimiento (GC) como propuesta para la colaboración en organizaciones inteligentes. Educar, 47 (1) No. 47, 1, 2011. Argentina (2003). Extraído de: http://dialnet. unirioja.es/servlet/articulo?codigo $=3696720$

Berengueras, M. El sistema educativo de Alemania (2012). En revista, http://www.adide.org/revista/index. php?option=com_content\&task=view\&id=266\&Itemid=71

Constitución Política de Colombia 1991. Extraído el 21 de octubre de 2014 de: http://www.senado.gov.co/ images/stories/Informacion_General/constitucion_politica.pdf

Constitución Política de Francia 1946. Preámbulo de la Constitución francesa de 1946, de 27 de octubre (la Constitución de la IV República). Journal Officiel de la République Française (en francés) (París): 9151-9173

CENEVAL, México D.F. Estructura del Sistema Educativo en México D.F. (2013), http://universidades-iberoamericanas.universia.net/mexico/sistema-educativo/estructura.html

Dretske, F. Conocimiento e información. Barcelona. España: Biblioteca Científica Salvat, 1987. Versión española de la obra original americana Knowledge \& Margarita Vicedo, Montserrat Guilla y Fina Pizarro.

Fajardo, C. Gerencia de organizaciones inteligentes. Innovar. Revista de ciencias administrativas y sociales (2011). Extraído de: http://www.bdigital.unal.edu.co/26593/1/24207-84758-1-PB.pdf

Ferrer, 0.T. Los procesos gerenciales en las organizaciones educativas desde el enfoque inteligente. Venezuela (2012). Extraído de: http://tesis.luz.edu.ve/tde_busca/arquivo.php?codArquivo=4485

Genesi, M. Gestión de calidad del talento humano en las organizaciones educativas inteligentes. Orbis: revistas de Ciencias Humanas No. 17. Venezuela (2010).

La France á la Loupe. El Sistema Educativo en Francia. Republica de Francia: Ministère des Affaires étrangères, 2007.

Lara, F.J. Estudio de la gestión del conocimiento sobre los resultado organizativos análisis del efecto mediador de las competencias directivas. España: Universidad Politécnica de Valencia, 2005. Extraído de: https:// riunet.upv.es/bitstream/handle/10251/1866/tesisUPV2414.pdf?sequence=24

León, R. Las organizaciones inteligentes. Vol. (6) 2. Lima, Perú: Industrial Data, 2003.

Maramotto, M.I. La gestión del conocimiento profesional en la dirección de instituciones educativas. España: Universidad Nacional de Educación a Distancia, 2005. Extraído de: http://dialnet.unirioja.es/servlet/ tesis? codigo $=37982$

Martínez, F.C. Gerencia de organizaciones inteligentes. En revista, Innovar, revista de ciencias administrativas y sociales No. f 5, Enero-Julio 2000.

Ministerio de Educación Nacional. Decreto Único Reglamentario del Sector Educación No. 1075. Mayo de 2015. Colombia.

Ministerio de Educación Nacional. Ley General de Educación en Colombia, 115 de 1994. Colombia.

Ministerio de Educación Nacional MEN. Proyecto de Modernización de las Secretarías de Educación. Extraído el 13 de octubre de 2014 de: http://www.modernizacionsecretarias.gov.co/index.php/proyecto/que-es 
Ministerio de Educación Nacional MEN. Proyecto Modernización de las Secretarias de Educación. Presentación (2014), http://www.modernizacionsecretarias.gov.co/

Ministerio de Educación del Ecuador. Nuevo Modelo de Gestión Educativa del Ecuador. Quito (2015). Extraído de: http://educacion.gob.ec/que-es-el-nuevo-modelo-de-gestion-educativa/

Muñoz, M.D. El largo camino hacia la gestión del conocimiento. Revista de Psicología del trabajo y de las organizaciones, vol. 19, núm. 2, 2003. Madrid. España. Extraído de: http://www.redalyc.org/articulo. oa?id=231318052005

Porras, E. Fundamentos básicos de gestión y calidad para instituciones educativas. Colombia: Limusa, 2012.

Ramos, J. (2008). La Educación un servicio público imprescindible para el progreso de la sociedad. Colombia. Aula de innovación Educativa.

Russell, S; Norving, P. Inteligencia artificial. Un enfoque moderno. Madrid: Prentice Hall, 1996. Traducido del inglés: Artificial Intelligence a modern approach.

Sarasara, C. Gestión del conocimiento y liderazgo de clase mundial para el desarrollo del capital intelectual en las organizaciones inteligentes. Perú, 2010.

Sánchez, M. La gestión del conocimiento y su relación con otras gestiones. Ciencias de la información. Vol. 37, No 2-3. Cuba. Ciudad Ángela (2006). Extraído de: http://www.redalyc.org/articulo.oa?id=181418190003.

Sander, B. Nuevas tendencias en la gestión educativa: democracia y calidad. $2^{\circ}$ Seminario de Actualización en Investigación Educativa. México: Universidad Marista, 2012.

Sañudo, L. Hacia un modelo de gestión del conocimiento educativo para instituciones de educación superior y centros de investigación. Congreso Iberoamericano de Ciencia, Tecnología, Innovación y Educación. Buenos Aires, Argentina, 2014.

SEP. Modelo de gestión educativa estratégica. Programa Escuelas de calidad México D.F. México (2015). Extraído de: http://basica.sep.gob.mx/pec/start.php?act=modelo.

Senge, P. La quinta disciplina. El arte y la práctica de la organización abierta al aprendizaje. Barcelona: Garnica, 1992.

Serlon, J. Conocimiento de la gestión de las organizaciones: Sistemas complejos dinámicos inestables adaptativos. España, 2010.

Tintoré, M. Las universidades como organizaciones que aprenden: el caso de la facultad de educación de la Universidad de Cataluña. España, 2010.

Torres, A y otros. Investigar en educación y pedagogía. Fundamentación y metodología. Facultad de Educación. Universidad de Nariño, 2002.

Valecillos, C.A. Modelos de gestión basado en enfoque de las organizaciones inteligentes para la Facultad de Ciencias Económicas y Sociales de LUZ. USA: Tecana American University, 2009.

Von Krogh, G. Making the most of your company's knowledge: a strategic framework. Long Range Planning, 34(4) (2001). Recuperado de http://www.redalyc.org/pdf/2313/231318052005.pdf

Unesco. World Data on education donnés mondiales de I_education, datos mundiales de educación. VII Ed. /11. 7ª Edición. IBE/2010/CP/cl. 2010.

Vázquez \& otros. Modelo de gestión educativa estratégica. Programa escuelas de Calidad Módulo I. Alianza por la calidad de la educación. Secretaría de Educación Pública Argentina 28, Colonia Centro Histórico, C.P. 06020, México, D.F., 2010. 